\title{
Self-Care on Hemodialysis: Behaviors With the Arteriovenous Fistula
}

\author{
Clemente Neves Sousa, ${ }^{1,2}$ Patrícia Marujo, ${ }^{3}$ Paulo Teles, ${ }^{4}$ Marta Nunes Lira, ${ }^{5}$ \\ and Maria Eulália Leite Mota Novais ${ }^{3}$
}

${ }^{1}$ Nursing School of Porto, Porto, Portugal and ${ }^{2}$ CINTESIS - Center for Health Technology and Services Research Faculty of Medicine, Porto University and ${ }^{3}$ Nursing School of Lisbon, Lisbon, Portugal and ${ }^{4}$ School of Economics and LIAAD-INESC Porto LA, Porto University, Porto, Portugal and ${ }^{5}$ SOS Renal Service, Olinda Pernambuco, Brasil and Nursing Master student, Federal University of Pernambuco, Recife, Brazil

\begin{abstract}
End stage renal disease (ESRD) patients should be educated to maintain and preserve the arteriovenous fistula (AVF) in the best condition. The purpose of this work was to evaluate self-care frequency and factors that influenced such frequency. A prospective study was performed in 101 hemodialysis patients. Self-care behaviors were measured with the Scale of Assessment of Self-Care Behaviours with Arteriovenous Fistula in Hemodialysis. A regression model was used to determine the relevant predictors of self-care frequency and their influence. The incidence of self-care behaviors was $71.0 \%$.
\end{abstract}

The regression model showed that self-care behaviors were positively influenced by gender (female), ESRD etiology (hypertension, polycystic kidneys and other kidney diseases), duration of AVF and negatively by the existence of previous AVF and health professional (doctor). The frequency of self-care behaviors was lower than expected and below an appropriate standard. Education programs designed to improve self-care behaviors with AVF should be further explored in a prospective randomized trial. Key Words: Arteriovenous fistula, Behaviors, Hemodialysis, Self-care, Vascular access.
Vascular access is very important for people with end stage renal disease (ESRD) and requires specific care. Arteriovenous fistula (AVF) is associated with fewer complications, lower morbidity, lower maintenance costs, extended patency and patient survival when compared with other vascular accesses. AVF optimization is an ongoing challenge for the interdisciplinary dialysis team (nephrologist, dialysis nurse and vascular surgeon) and patient. Education of the dialysis patient is an essential element to improve health care quality (1).

Several vascular access guidelines recommend that patients should be educated concerning vascular access care (2-4). Patients should develop selfcare behaviors with their AVF in order to maintain it in the best condition, because its state influences the effectiveness of the dialysis treatment (5). During pre-dialysis/dialysis, it is essential to educate patients

Received September 2016; revised November 2016.

Address correspondence and reprint requests to Clemente Neves Sousa, PhD, RN, Nursing School of Porto, Rua Dr. António Bernardino de Almeida, 4200-072 Porto, Portugal. Email: clementesousa@esenf.pt about AVF, which can lead to an increase in the number of patients performing hemodialysis (HD) through fistula at dialysis initiation $(6,7)$.

When information about vascular access is provided to patients, an improvement in access self-care is achieved (8) and consequently patients have better care with their fistula. Little is known about the relationship between patients' self-care with AVF and self-care frequency. We do not know the proportion of patients who are able to take care of their AVF or the proportion of those who are not and need to be educated. Although self-care with AVF has long been recognized as important, little is known about the care actually performed by patients (2-4). Our study was designed to evaluate the frequency of self-care behaviors with AVF and to uncover the variables that affect its frequency.

\section{PATIENTS AND METHODS}

\section{Study Design}

This was a prospective and observational study in a dialysis unit involving patients who used an AVF for 
HD. The study started after approval by the institution ethics committee.

\section{Study Setting and Population}

The study was carried out in a private dialysis unit in the Lisbon region, Portugal. The criteria for participants to be eligible for the study were to be 18 years or older, an AVF more than 6 months old on HD, absence of memory problems and medically stable. Participants were invited to participate in the study. Participants with no interpretation problems completed the questionnaire autonomously and those with problems were assisted by the researcher. A total of 101 patients were included and 10 patients refused to participate in study.

\section{Data Collection and Instrument}

All data were collected between January and February 2016. Information concerning demographic characteristics (age, gender, education, employment, marital status) and clinical characteristics (ESRD etiology, dialysis vintage, previous AVFs, AVF duration, information about care with the AVF) was collected from a questionnaire designed by the authors. Information concerning self-care behaviors with the AVF was collected from the Scale of Assessment of Self-Care Behaviours with Arteriovenous Fistula in Hemodialysis (ASBHD-AVF) (8). This scale has 16 items in two subscales: prevention of complications (10 items) and management of signs and symptoms (6 items) (8). Responses to each item are based on a Likert scale with five possible answers. Higher scores indicate patients' higher frequency of self-care with the AVF.

\section{Statistical Analysis}

Quantitative variables were described by the mean and standard deviation (SD). The distribution of categorical variables was represented by proportions.

A regression model was applied to each sub-scale and the estimation results include parameter estimates, $P$-values and $95 \%$ confidence intervals (CIs). The global scale can be unable to uncover the different patterns exhibited by the two sub-scales. We chose to analyze each sub-scale separately. The independent variables considered were gender, age, education, employment, marital status, dialysis vintage, diabetes, hypertension, polycystic kidney, other kidney diseases, infections disease, autoimmune disease, previous AVFs, AVF duration and information about care with AVF. A 5\% $P$-value was considered statistically significant. Analysis was performed with the Statistical Package for the Social Sciences Software (version 23.0; IBM Co., Armonk, NY, USA) and with the language $\mathrm{R}$ (version 3.3.1, R Foundation for Statistical Computing, Austria).

\section{RESULTS}

\section{Patient Characteristics}

This study involved 101 patients with AVF on HD treatment (Table 1). Two-thirds of the patients were male $(66.3 \%)$. Concerning sociodemographic characteristics, the mean age was 60.9 years (with a SD of 13.4), most ages ranged between 50 and 70 years and there were few patients less than 50 years old. The proportion of married patients was $51.4 \%$, $39.6 \%$ of the patients had 4 years' education and $20.8 \%$ had a college education. Most of the patients (58.4\%) were retired.

Concerning clinical characteristics of the study, $26.7 \%$ had hypertension and $52.5 \%$ had more than 5 years HD treatment. The fistula duration mean was 71.3 months (with a SD of 78.3 ; range: 6-432 months), but the median time was 48 months

TABLE 1. Global characterization of the sample

\begin{tabular}{|c|c|}
\hline & All patients $(N=101)$ \\
\hline Male/Female (\%) & $66.3 / 33.7$ \\
\hline Age, years $($ mean $\pm S D)$ & $60.9 \pm 13.4$ \\
\hline \multicolumn{2}{|l|}{ Marital status (\%) } \\
\hline Married & 51.4 \\
\hline Divorced & 8.9 \\
\hline Widowed & 12.9 \\
\hline Single & 26.8 \\
\hline \multicolumn{2}{|l|}{ Education (\%) } \\
\hline$\leq 4$ years & 39.6 \\
\hline $5-9$ years & 19.8 \\
\hline $10-12$ years & 19.8 \\
\hline College & 20.8 \\
\hline \multicolumn{2}{|l|}{ Employment (\%) } \\
\hline Employed & 27.7 \\
\hline Unemployed & 12.9 \\
\hline Retired & 58.4 \\
\hline Student & 1.0 \\
\hline \multicolumn{2}{|l|}{ ESRD etiology (\%) } \\
\hline Diabetes mellitus & 6.9 \\
\hline Arterial hypertension & 26.7 \\
\hline Polycystic kidney & 12.9 \\
\hline Autoimmune disease & 6.0 \\
\hline Glomerular & 11.9 \\
\hline Other kidney disease & 35.6 \\
\hline \multicolumn{2}{|l|}{ Dialysis vintage (\%) } \\
\hline$\leq 1$ years & 9.9 \\
\hline $1-5$ years & 37.6 \\
\hline$\geq 5$ years & 52.5 \\
\hline AVF duration (months) (mean $\pm \mathrm{SD})$ & $71.3 \pm 78.3$ \\
\hline \multicolumn{2}{|l|}{ Number previous AVFs (\%) } \\
\hline 1 & 74.4 \\
\hline 2 & 20.4 \\
\hline$\geq 3$ & 5.2 \\
\hline \multicolumn{2}{|l|}{ Information about care with AVF (\%) } \\
\hline No one & 15.8 \\
\hline Doctor & 4.0 \\
\hline Nephrologist & 50.5 \\
\hline Nurse & 28.7 \\
\hline Other patients & 1.0 \\
\hline
\end{tabular}

AVF, arteriovenous fistula; ESDR, end stage renal disease; $\mathrm{SD}$, standard deviation. 
(i.e., $50 \%$ of the patients had the fistula for 48 months or less). A small number of patients had a previous fistula $(25.6 \%)$ and $88.2 \%$ of them had an access loss caused by thrombosis. A patient had four previous fistulas $(2.6 \%)$ and $20.4 \%$ had two. The information received by the patients about the care with fistula was provided by the nephrologist $(50.5 \%)$, the nurse $(28.7 \%)$, the doctor $(4 \%)$, or no one $(15.8 \%)$.

\section{Self-Care Behaviors}

Descriptions of fistula self-care behaviors for the global scale and both sub-scales are provided in Figure 1.

The proportion of patients who performed self-care behaviors with fistula was $71.0 \%$, (with a SD of 13.6), but there is a considerable number of patients with low or very low frequencies (the minimum is $28.1 \%$ only). Concerning the management of signs and symptoms sub-scale, high and very high frequencies are strongly predominant. In fact, very few patients have low frequencies and consequently there is a strong concentration in the high and very high frequencies, reflected in the mean value of $82.8 \%$, a large value (with a SD of 18.1). On the contrary, the moderate frequencies in the central part of the distribution dominate the prevention of complications sub-scale. Nevertheless, there is an important number of patients with low or very low frequencies or with high frequencies, whereas only a small number has very high frequencies. Consequently, the mean is only $63.9 \%$ (with a SD of 16.9), a moderate value, much lower than the mean of the other sub-scale.

Regression analysis (Tables 2 and 3 ) shows that the frequency of self-care behaviors in management of signs and symptoms sub-scale increases with the fistula duration (0.039, 95\% CI:-0.003; 0.082), is lower for patients with a previous fistula $(-7.99,95 \% \mathrm{CI}:-14.78$; -1.21) and for patients whose information was
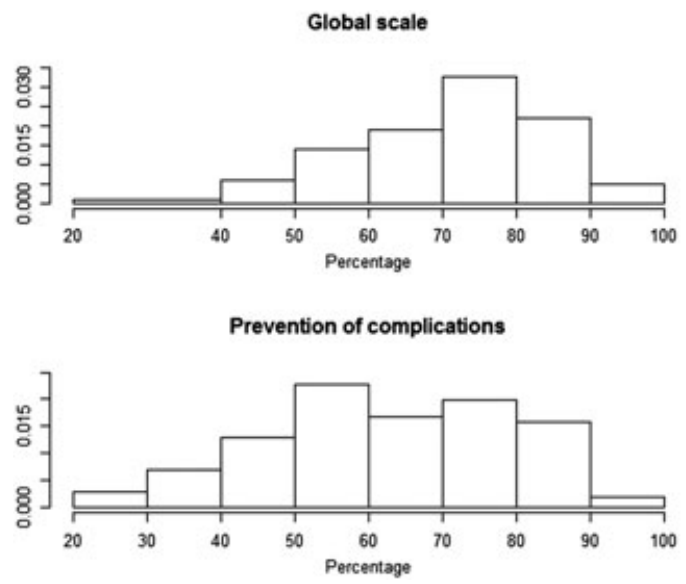

TABLE 2. Regression model for the management of signs and symptoms sub-scale

\begin{tabular}{lrcc}
\hline & \multicolumn{3}{c}{ Estimated parameters } \\
\cline { 2 - 4 } Variable & Estimate & $P$-value & $95 \%$ CI \\
\hline AVF duration & 0.039 & 0.069 & {$[-0.003,0.082]$} \\
Previous AVF & -7.998 & 0.021 & {$[-14.786,-1.209]$} \\
Doctor & -29.779 & 0.001 & {$[-46.743,-12.816]$} \\
\hline
\end{tabular}

CI, confidence interval.

TABLE 3. Regression model for the prevention of complications sub-scale

\begin{tabular}{lrcc}
\hline & \multicolumn{3}{c}{ Estimated parameters } \\
\cline { 2 - 4 } Variable & Estimate & $P$-value & $95 \% \mathrm{CI}$ \\
\hline Female & 9.274 & 0.006 & {$[2.777,15.770]$} \\
Hypertension & 14.856 & 0.005 & {$[4.499,25.213]$} \\
Polycystic kidney & 13.213 & 0.032 & {$[1.176,25.251]$} \\
Other kidney disease & 11.397 & 0.026 & {$[1.367,21.427]$} \\
Doctor & -26.232 & 0.002 & {$[-42.289,-10.174]$} \\
\hline
\end{tabular}

CI, confidence interval.

provided by the doctor $(-29.78,95 \%$ CI: -46.74 ; -12.82). Concerning the prevention of complications sub-scale, the frequency of self-care behavior is higher for women $(9.27,95 \% \mathrm{CI}: 2.77 ; 15.77)$, for hypertension patients (14.85, 95\% CI: 4.49;25.21), for patients with polycystic kidneys (13.21, 95\% CI: 1.76;25.25) and for patients with other kidney disease $(11.39,95 \% \mathrm{CI}$ : $1.36 ; 21.42)$, and is lower for patients whose information was provided by the doctor $(-26.23$, 95\% CI: -42.29; -10.17).

\section{DISCUSSION}

The literature shows few studies on the evaluation of self-care behaviors with AVF by the patient. Most

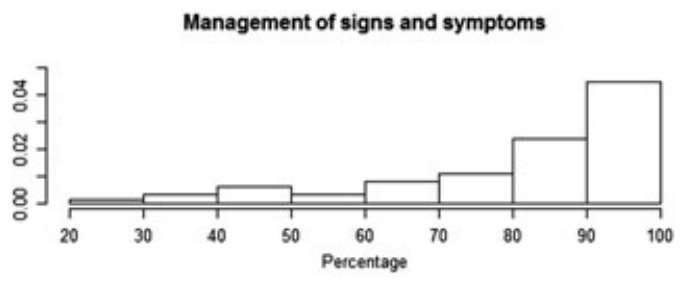

FIG. 1. Distribution of Self-care Behaviours with Arteriovenous Fistula (AVF) (global scale and sub-scales). 
are studies based on a qualitative methodology and do not assess the frequency of self-care behaviors but only which self-care behaviors are performed by patients. Our study assessed that frequency and identified the variables that affect it.

The frequency of self-care behaviors with AVF by patients was $71.0 \%$, which is lower than expected and below an appropriate standard. Futhermore, some patients showed a frequency of self-care behaviors a little above $25 \%$ only and none of the patients performed all self-care behaviors. Patients in our sample exhibited less self-care behaviors with AVF when compared with the scale validation study, where the frequency of such behaviors was $82.4 \%$ (minimum of $21.9 \%$; maximum of $100 \%$ ) (8). In another study with 30 ESRD patients on HD, 97.7\% had inadequate self-care behaviors with AVF (9). There are strong differences in self-care behaviors between both studies.

In a qualitative study with 26 ESRD patients on HD, participants say that they had to be careful with situations that could damage vascular access, particularly knocks or shocks with the fistula arm (10). In a group with 21 ESRD patients on HD, 13 patients avoided trauma in the fistula arm (11). In another study, $43.3 \%$ patients avoided shocks in the fistula arm (9). In our study, $82.8 \%$ of the patients showed a good self-care with AVF performance associated with management of signs and symptoms. It will be important in future studies to investigate which aspects do influence patients to perform certain self-care behaviors.

Concerning the prevention of complications, selfcare behaviors that aim at preventing infection and thrombosis are associated. In our study, the frequency of these self-care behaviors was only moderate, just over $60 \%$. In other studies, the frequencies of self-care behaviors were lower than in our study $(9,11)$. For example, in a sample of 30 ESRD patients on HD, two $(6.7 \%)$ patients said that they felt the thrill of the fistula (9) or in another study only one patient out of 21, evaluated the thrill (11). The thrill of the fistula is an important means to check how the vascular access is working at any particularly time, allowing early thrombosis identification (12). It will be important in the future to develop retrospective studies in order to investigate the factors that can influence self-care behaviors with AVF in the prevention of complications.

One of the most relevant findings of this study was the identification of the variables that affect the frequency of self-care behaviors with AVF. AVF duration, previous AVF and the doctor as information provider influence self-care behaviors in management of signs and symptoms. The frequency of self- care increases 0.039 percentual points (p.p.) for each additional month after fistula creation. The frequency of self-care for patients with a previous fistula is 8 p.p. lower than that of patients with no previous fistula. The frequency of self-care for patients whose information was provided by the doctor is approximately 30 p.p. lower than that of patients with any other source of information.

The frequency of self-care behaviors in the prevention of complications for females is 9.3 p.p. higher than for males. The frequency of self-care for hypertension patients is 14.9 p.p. higher than that of patients with diabetes or with autoimmune disease or with infectious disease who have a similar frequency. The frequency of self-care for patients with polycystic kidneys is 13.2 p.p. and other kidney disease is 11.4 p.p. higher than that of patients with diabetes or with autoimmune disease or with infections disease who have a similar frequency. We have not found any study in the literature concerning the identification of variables affecting the frequency of selfcare behaviors with AVF. It is necessary to conduct more research studies about this topic to confirm if these variables do affect the frequency of self-care behaviors and if other variables are also relevant.

The implementation of patient education programs is an appropriate strategy to increase self-care behaviors with AVF. Such programs are recognized as essential to empower patients, to raise the level of knowledge and skills/self-care behaviors about vascular access $(1,10)$. A study with 223 ESRD patients in stages 4 and 5 has shown that patients have a tendency to improve self-care with vascular access when information is provided by a nurse (11). However, those programs should be continuous, multidisciplinary, structured and also tailored to patient goals and preferences (1). A qualitative experimental study demonstrated that education from patient to patient has more influence than when provided by the healthcare professional (13). Therefore, experimental education should be considered in such programs.

The dialysis unit team (nephrologist, dialysis nurse and doctor) needs to improve the strategy of patient education about vascular access in order to increase the frequency of patients' behavior for all patients, but especially for those with lower frequency of self-care behaviors.

\section{CONCLUSION}

We concluded from the fitted model that the frequency of self-care behaviors with arteriovenous fistula is positively influenced by being a female, being hypertensive, having polycystic kidneys, 
having other kidney disease and arteriovenous fistula duration, and is negatively influenced by previous arteriovenous fistula and the doctor as information provider about the fistula. These were the only variables that significantly affected the frequency of self-care behaviors among all the independent variables considered in the analysis. The self-care behaviors with fistula were performed in $71.0 \%$ of the patients. Education programs designed to improve self-care behaviors with arteriovenous fistula should be further pursued in a prospective randomized trial, in an effort to better identify the factors that influence self-care with fistula.

Conflicts of Interest: The authors do not have any conflict of interest and were not funded.

\section{REFERENCES}

1. Moist LM, Lee TC, Lok CE et al. Education in Vascular Access. Semin Dial 2013;26:148-53.

2. NKF-K/DOQI, Kidney Disease Outocomes Quality Initiative. Clinical practice guidelines for vascular access: update. Am J Kidney Dis 2006:48:S176-S276.

3. Tordoir J, Canaud B, Haage P et al. EBPG on Vascular Access. Nephrol Dial Transplant 2007;22:ii88-ii117.
4. Kukita K, Ohira S, Amano I et al. 2011 update Japanese Society for Dialysis Therapy Guidelines of Vascular Access Construction and Repair for Chronic Hemodialysis. Ther Apher Dial 2015;19:1-39.

5. Sousa CN, Apostolo JL, Figueiredo MH, Martins MM, Dias VF. Interventions to promote self-care of people with arteriovenous fistula. J Clin Nurs 2014;23:1796-802.

6. Bowling $\mathrm{CB}$, O'Hare AM. Managing older adults with CKD: individualized versus disease-based approaches. Am J Kidney Dis 2012;59:293-302.

7. Lacson E Jr, Wang W, DeVeries C et al. Effects of a nationwide predialysis educational program on modality choice, vascular access, and patient outcomes. Am J Kidney Dis 2011;58:235-42.

8. Sousa CN, Apostolo JL, Figueiredo MH, Dias VF, Teles P, Martins MM. Construction and validation of a scale of assessment of self-care behaviors with arteriovenous fistula in hemodialysis. Hemodial Int 2015;19:306-13.

9. Pessoa NRC, Linhares FMP. Hemodialysis patients with arteriovenous fistula: knowledge, attitude and practice. Esc Anno Nery 2015;19:73-9.

10. Taylor MJ, Hanson CS, Casey JR, Craig JC, Harris D, Tong A. "You know your own fistula, it becomes a part of you"-Patient perspectives on vascular access: A semistructured interview study. Hemodial Int 2016;20:5-14.

11. Furtado AM, Lima FET. Autocuidado dos pacientes portadores de insuficiência renal crônica com a fístula artério-venosa. Rev Gaúcha Enferm 2006;27:532-8.

12. Sousa CN, Apostolo JL, Figueiredo MH, Martins MM, Dias VF. Physical examination: How to examine the arm with arteriovenous fistula. Hemodial Int 2013;17:300-6.

13. Xi W, Harwood L, Diamant MJ et al. Patient attitudes towards the arteriovenous fistula: a qualitative study on vascular access decision making. Nephrol Dial Transplant 2011;26:3302-8. 